\title{
Research on Gender Differences in Autism: From Genes, Sex Hormones to Brain Structures
}

\author{
Xuemei Liang \\ The Four Affiliated Hospital of Qiqihar Medical College, Heilongjiang Qiqihar 161000, China
}

Keywords: gender differences, Autism, genes, sex hormones, brain structures

\begin{abstract}
In autism spectrum disorders, the proportion of males is much higher than that of females, and there is a difference in male and female autism. In this paper, the factors affecting autism than gender differences are combed from the perspectives of gene, sex hormones and brain structure. Genetic studies have shown that autism genes have a "female protection effect", and male autistic patients are more likely to be more sensitive to males' risk genes for autism. Sex hormone studies suggest that differences in the effects of testosterone and sex hormones on brain development in men and women may be responsible for the gender differences in autism. Brain structure studies further reveal the specific manifestations of this difference and its relationship with autism. Future research needs to use a variety of methods to study the genetic, endocrine and brain structure differences of individuals with autism and their parents.
\end{abstract}

\section{Introduction}

Autism (ASDs, also known as autism) is a widespread developmental disorder caused by abnormal brain development. Its core characteristics include social communication barriers, speech and non-verbal development barriers, and repeated stereotypes and limited hobbies. It is a lifelong obstacle. In recent years, the incidence of autism has been on the rise. For example, in the United States and Britain around 1999, the incidence of autism was roughly 10 / 10000. Ten years later, the incidence of autism in the UK reached 157 / 10000, and the United States reached 113 / 10000 in 2012. If we look at the incidence of autism in school-age children (6 - 17 years old) alone, the United States in 2013 was as high as 2/100. Japan's research survey in 1996 showed that the incidence of autism in the country was 21. 1 / 10000, and by 2008, its incidence has increased dramatically to 181 / 10000. So far, the pathogenesis of autism remains unclear. The researchers speculate that the emergence of the disease is mainly related to genetic (chromosomal variation, gene mutation and copy number variation) and environmental (prenatal viral infection, sex hormone abnormalities, parental age, etc.) interaction. After more than 70 years of tortuous research, although humans have a relatively clear exploration of the causes of autism, the current clinical diagnosis of autism is still only at the behavioral level.

The incidence of autism is higher in men than in women, which has formed a consensus among researchers in countries around the world. In 1943, Leo Kanner first published 11 cases of autistic children, up to 8 male patients, only 3 female patients, and the children with Asperger's disease described in the report were male. Subsequent extensive studies have also found gender differences in the incidence and clinical manifestations of autism.

The incidence of ASD worldwide is about 7.6\%. Studies have shown that although the prevalence of men and women gradually decreased from 5:1 in 1995 to 3.1:1 in 2010, men are still the mainstay of autistic patients. Therefore, the number of male autistic patients is much more than that of females is a prominent phenomenon in autism research. In addition, there are differences in behavioral performance between patients with amphoteric autism. Male autistic patients show more explicit behavioral problems, such as violations, hyperactivity, more stereotyped behavior, and narrower interests. Female autistic patients have more severe internalization symptoms such as anxiety, depression, social problems, attention problems and thinking problems. There is no gender difference in childhood core autism symptoms, but female autistic patients have more sensory impairments during their lifetime. In terms of information processing, some studies suggest that for 
non-social information, male processing ability is stronger than female, that is, male systemic ability is stronger, and for social information processing, women have more advantages and performance is stronger. When comparing the male-female differences within the autistic patient group, the empathy ability was found. The autistic women scored higher than the autistic men, while the systemic ability autism men scored higher than the autistic women. These findings suggest that there is a significant gender difference in autism. Past research and clinical literature is based on a male orientation to describe autism, and mentioning female autism to the same height will help us understand and intervene in autism. In addition, gender influence factors are also an important perspective in constructing the conceptual framework of autism. This paper intends to explore the gender differences in the behavioral gender differences and incidence ratios of autism from the perspectives of gender differences in gender, endocrine gender differences, and gender differences in brain neural structure.

\section{Gender Differences in Children with Autism}

The incidence of autism is significantly higher in men than in female autistic children. The proportion of male patients is higher than that of female patients. Fombonne analyzed 43 literatures on the incidence of autism since 1966 and found that the male-female ratio of autistic children was roughly 1 . Among them, the ratio of male to female in typical autism (ASD) is 4. 3: 1 or so, in those autistic children without mental retardation, the ratio of male to female is 5 . This shows that IQ may be an important factor affecting the sex ratio of autism. With the gradual increase of IQ level, the male-female ratio of the incidence of autistic children is gradually increasing. In those children with high-functioning autism with relatively high IQ, the male-female ratio is much higher than that of IQ. This was confirmed by related surveys. The study found that in low-function autism $(\mathrm{I}<55)$, the sex ratio of male to female morbidity was 2. 5: 1 - 3: 1 or so, and in Asperger's disease, the ratio of male to female incidence of this disorder is around 6: 1 - 16: 1 .

The three core characteristics of autism have gender differences and are influenced by individual function and brain neural mechanism. Social interactions: Gender differences in performance areas In terms of social interaction, researchers examined the gender differences in children with high-functioning autism and low-function autism. The results showed that girls in the Highly Functional Autism Group (HFA) had lower communication and social skills than boys. For example, after comparing 42 autistic children (21 males and females) with IQ values greater than 60, McLennan found that the number and quality of girls' friendships were significantly lower than boys in high-function autism (HFA) samples. There were no significant differences in other areas; Banach et al. also found that girls' communication skills and adaptive behavior showed more damage than boys. However, Baron-Cohen et al. compared 65 males and 25 female high-functioning autistic patients (HFA) and found that the two showed the opposite difference in empathy ability, that is, autistic girls were significantly better than boys. In the study of children with low-functioning autism (LFA), the above differences are not obvious. In addition, in social behavior, young autistic boys show more tendency to attack and hyperactivity, while autistic girls tend to show more social withdrawal tendency.

Speech and non-verbal development: Men and women are roughly similar, but there are some differences in the development of speech and non-verbal. Most of the research results show that the gender difference is not obvious. For example, Marjorie et al. compared 40 people aged 8-18. ASD children (20 males and 20s, including high-functioning autism, Asperger's disease and PDD-NOS) and 36 typical autistic children (TYP, 19 girls, 17 boys) were found in In terms of language use, men and women do not differ in speech, grammar, semantics, context, coherence, consistency, and initiation. In terms of non-verbal communication, Park et al. compared 91 boys and 20 girls after matching age and IQ and found that the level of development of boys' nonverbal communication was significantly higher than that of girls.

Repetitive behavior and abnormal interest: Boys have more problems than girls in terms of repetitive behavioral anomalies, and Hartley et al. used the Achenbach Child Behavior Scale (CBCL) for ages of 1.5 - 3. A comparative study of 157 boys and 42 girls between the ages of 9 
found that boys were more serious than girls in terms of repetition and stereotypes, except for the problem of boys with limited interest. After strict matching of age and IQ, Holtmann et al. also used CBCL for 23 autistic children aged 5-20 years, and found that Sigan and the consistent conclusion that autistic boys are repeating behaviors and abnormalities. Interest is indeed more serious than the girl problem.

There may be differences in the neurological mechanisms of autistic children. In recent years, with the rapid development of brain imaging techniques (MRI, PET and ERP, etc.), experimental research on cognitive processing in children with autism is increasing. At present, the main areas of concern of researchers include memory, face recognition, speech language processing, executive function, theory of mind and emotional cognition. Some studies have initially revealed the differences in the cranial nerve mechanisms of cognitive processing in autistic boys and girls: At rest, the default mode network (DMN) of men in the normal population is lower than that of females. In autistic patients, this functional connectivity of men are even lower; in the bilateral subgingival area, which is highly correlated with emotions, male autistic patients have more activity when performing emotional recognition tasks. It is also shown to be significantly lower in women with autism.

Based on the above findings, we found that: (1) children with autism have significant gender differences in morbidity and are regulated by intelligence levels; (2) gender differences in clinical manifestations in children with autism; (3) loneliness There are differences in the neural mechanisms of the brain in children with symptoms.

\section{Influencing Factors of Gender Differences in Autism}

In the process of exploring autism genes, the researchers found that although autistic women have more pathogenic genes, their symptoms are much milder than men's. Therefore, the researchers proposed the Female Protec-tive Model, which suggests that despite the number of gene copy variations in female autistic patients, the number of mononucleotide variants is much higher than that of male autistic patients, ie females. There are many high-risk genetic mutations associated with autism; however, women do not show autism symptoms, because the appearance of female autism autosomal genetic stalk gene deletion is reduced compared with men. Studies have shown that even if the genetic risk of autism in the family is high, the protective effect of women will still work: if the first child in a family is a girl and suffers from autism, the risk of the next child is higher than those of the head. The fetus is a boy and a sick family. The relationship between sex hormones and autism is more complicated. Studies have found that the content of androgen in autistic men is positively correlated with the severity of the disease. However, studies by Beje-rot et al. have shown that the female traits of male autistic patients are more pronounced than those of the control group, while the male traits of female autistic patients are more pronounced than those of the control group. The neuroendocrine results associated with this indicate that there is a separation between autistic men and women, and that women with autism have higher levels of androgens than normal women, while male androgen are lower than normal men. Abnormalities in these hormone levels may be responsible for the abnormalities of the systemic-sympathetic ability of autistic patients.

Sex hormones have a certain regulatory effect on the development of the individual, so in the future, sex hormones may be a new means to block the specific tendency of individual loneliness genes. To achieve this goal, it is necessary to clarify the interaction between testosterone and the neurodevelopmental pathway, and to increase the risk of autism, and to clarify the specific state of the cellular and molecular mechanisms in this process.

The longitudinal study of brain structure in autism shows that the development curve of the whole brain volume of children with autism is initially rising, gradually decreasing at the beginning of puberty, and finally coincides with the development curve of normal children at 10-15 years old. Many other brain structures in autistic patients continue to decline abnormally during puberty. From childhood to adolescence, with the changes of time, the whole brain volume and local brain volume of autistic patients will undergo complex dynamic changes 
The neuroanatomical findings of children with autism indicate that the gray matter in the middle of the left occipital gyrus and the right anterior temporal gyrus of the boy with autism is larger than that of the normal boy; the bilateral frontal lobe of the autistic girl (bilateral Frontal Lobe) The gray matter volume is significantly larger than that of normal girls. The neuroanatomical differences between autistic boys and girls are mainly due to the girl's right inferior Cin-gulate Gyrus and right cerebellum gray matter. The volume is larger than the boy. Men have larger cerebrospinal fluids with more gray matter and white matter than men.

\section{Conclusion}

At present, there is a large amount of evidence that there is a close relationship between gender factors and the onset of autism. The important role of sex hormones in the pathogenesis of autism is also increasingly valued by researchers. With the deepening of relevant research, the specific biological mechanism of sex hormones affecting autism is constantly being elucidated. I believe that these tasks will definitely help the diagnosis, treatment and prevention of autism.

\section{References}

[1] Fred RV, Lisa AW. A practical guide to autism-what every parent,family,and teacher needs to know [J]. Hoboken: John Wiley \&Sons, 2009: 1 - 24.

[2] Fombonne E. Epidemiology of pervasive developmental disorders [J]. Pediatr Res, 2009, 65(6): 591 - 598.

[3] Fan Yuebo, Jie Xiaofeng, Zou Xiaobing. Review of the prevalence of autism [J]. Chinese Journal of Child Health, 2008, 16(4): 439 - 440.

[4] Harony H,Wagner S. The contribution of oxytocin and vasopressinto mammalian social behavior: potential role in autism spectrumdisorder[J]. Neurosignals, 2010, 18(2): 82 - 97.

[5] Rutter ML. Progress in understanding autism: 2007 - 2010 [J]. JAutism Dev Disord, 41(4): 395 404. 